\title{
The Effect of Scalp Point Cluster-Needling on Learning and Memory Function and Neurotransmitter Levels in Rats with Vascular Dementia
}

\author{
Junli Yang, ${ }^{1}$ Gerhard Litscher, ${ }^{1,2}$ Haitao Li, ${ }^{1}$ Wenhai Guo, ${ }^{1}$ Zhang Liang, \\ Ting Zhang, ${ }^{1}$ Weihua Wang, ${ }^{1}$ Xiaoyan Li, ${ }^{1}$ Yao Zhou, ${ }^{1}$ Bing Zhao, ${ }^{1}$ Qi Rong, \\ Zemin Sheng, ${ }^{1,3}$ Ingrid Gaischek, ${ }^{2}$ Daniela Litscher, ${ }^{2}$ and Lu Wang ${ }^{1,2}$ \\ ${ }^{1}$ Second Affiliated Hospital of Heilongjiang University of Chinese Medicine, Harbin 150001, China \\ ${ }^{2}$ Research Unit for Complementary and Integrative Laser Medicine, Research Unit of Biomedical Engineering in \\ Anesthesia and Intensive Care Medicine, and TCM Research Center Graz, Medical University of Graz, 8036 Graz, Austria \\ ${ }^{3}$ Privatclinic Lassnitzhoehe, 8301 Lassnitzhoehe, Austria
}

Correspondence should be addressed to Junli Yang; yul2004jp@yahoo.co.jp and Gerhard Litscher; gerhard.litscher@medunigraz.at Received 26 April 2014; Revised 1 June 2014; Accepted 2 June 2014; Published 15 June 2014

Academic Editor: Yi-Hung Chen

Copyright (C) 2014 Junli Yang et al. This is an open access article distributed under the Creative Commons Attribution License, which permits unrestricted use, distribution, and reproduction in any medium, provided the original work is properly cited.

\begin{abstract}
We observed the effect of scalp point cluster-needling treatment on learning and memory function and neurotransmitter levels in rats with vascular dementia (VD). Permanent ligation of the bilateral carotid arteries was used to create the VD rat model. A Morris water maze was used to measure the rats' learning and memory function, and the changes in neurotransmitter levels in the rats' hippocampus were analyzed. The results show that scalp point cluster-needling can increase the VD rat model's learning and memory score. The VD rat model's learning and memory score was significantly different when compared with that of the sham operation group $(P<0.05)$. Hippocampal acetylcholine (ACh), dopamine (DA), and 5-hydroxytryptamine (5-HT) concentrations significantly decreased in the rat model. Compared with the model group, the scalp point cluster-needling group's ACh concentration markedly increased and DA and 5-HT levels increased as well. In conclusion, scalp point cluster-needling can improve learning and memory function in VD rats, and its function may be related to an increase in neurotransmitter release.
\end{abstract}

\section{Introduction}

Vascular dementia (VD) is an acquired intellectual impairment due to impairment in brain function caused by cerebrovascular disease. It is a chronic progressive disease and its main manifestation is changes in learning and memory $[1,2]$. As the population gets older and cerebrovascular disease prevalence increases, the occurrence of VD increases as well. In Asian countries, VD accounts for $68.5 \%$ of dementia in the older population. In China, it accounts for $60-70 \%$, taking first place [3]. It is predicted that, by 2040 , the number of people with dementia worldwide will reach 81.1 million, with the number of dementia patients in China being the sum of those in all the developed countries combined [4]. VD not only severely damages physical and mental health, but also is a serious financial and mental burden on society and the family of those affected. Therefore, it has caught the widespread attention of society and the medical community at home and abroad [5].

Scalp point cluster-needling is a new technique based upon the summation of acupuncture expert Professor Yu Zhishun's many years of clinical experience and is used to treat cerebral infarction. It has been practically proven that scalp point cluster-needling is effective in treating cerebral infarction $[6,7]$. The effectiveness of using scalp point clusterneedling to treat VD has yet to be reported. This experiment aims to observe the effect of scalp point cluster-needling on learning and memory function and neurotransmitter levels in rats with VD. Also, this paper will discuss the clinical 


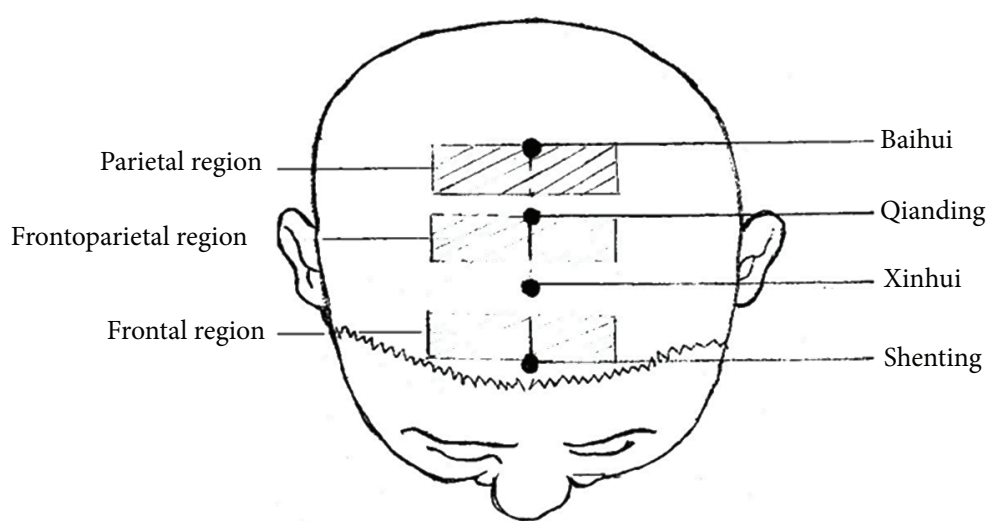

FIGURE 1: Cluster-needling (note: frontal region: Shenting penetrating Baihui and parallel from Qucha to Benshen. Directly below is the front area of the frontal lobe; parietal region: Baihui penetrating Qianding and 1 cun lateral to right and left Sishencong. Directly below is the precentral gyrus, postcentral gyrus, paracentral lobule, and a portion of the superior parietal lobule and inferior parietal lobule; frontoparietal region: Qianding penetrating Xinghui and Tongtian penetrating Chengguang and Zhengying penetrating Muchuang on both sides. Directly below is the superior frontal gyrus and posterior portion of the middle frontal gyrus).

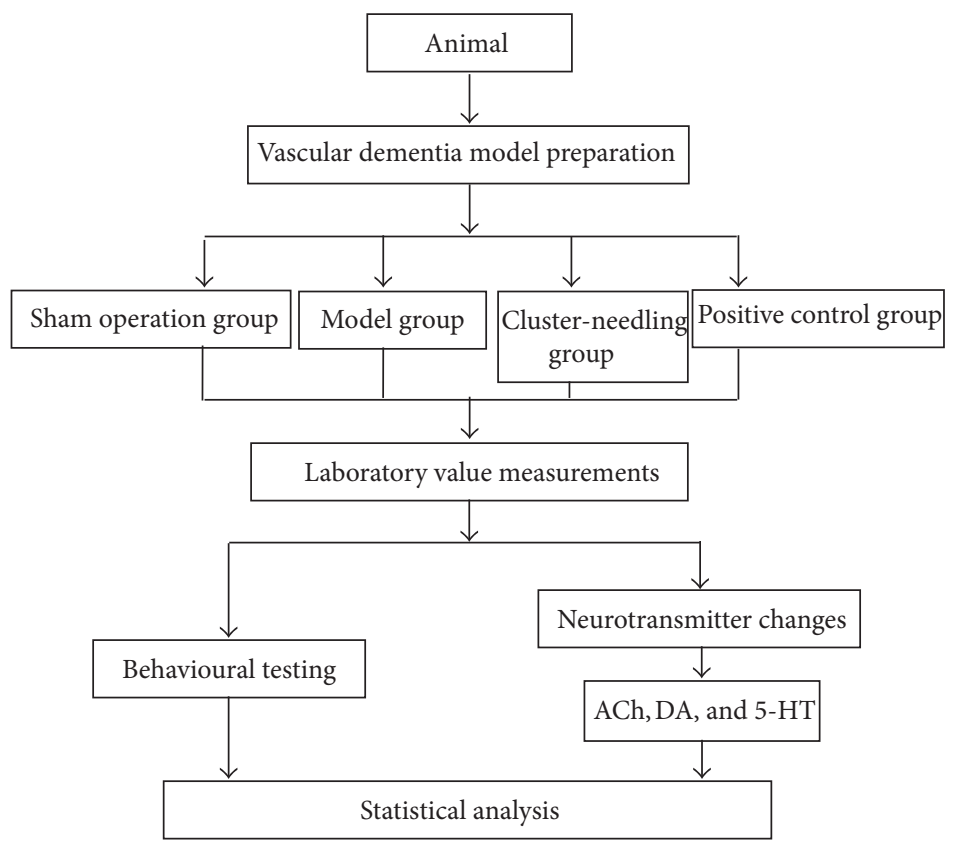

FIGURE 2: Flowchart of the experimental study.

effectiveness and possible mechanisms of scalp point clusterneedling in treating $\mathrm{VD}$, thereby providing a new train of thought for the prevention and treatment of $\mathrm{VD}$.

\section{Materials and Methods}

2.1. Animals. 75 Wistar rats (half female, half male, weighing 200-250 g each) were provided by the Heilongjiang University of Chinese Medicine Experimental Animal Center (experimental animal use permit no. SYXK Hei 2008001). They were housed under standard rearing techniques, and room temperature was maintained at $20-25^{\circ} \mathrm{C}$. The experiment started after 7 days of adaptive feeding. The investigations were carried out in compliance with the institutional animal care and use committee guidelines.

2.2. Main Apparatus. The Morris water maze was manufactured by Anhui Huaibei Zhenghua Biological Instruments and Equipment Ltd., the RF-5301PC fluorescence spectrophotometer by Shimadzu Japan, and the Huatuo brand acupuncture needles $(0.30 \mathrm{~mm} \times 13 \mathrm{~mm})$ by Suzhou Medical Appliance Factory Ltd. 
TABLE 1: Different treatment groups and interventions.

\begin{tabular}{lcccc}
\hline & Sham operation group & Model group & Positive control group & Cluster-needling group \\
\hline Carotid artery ligation & - & $\mathrm{X}$ & $\mathrm{X}$ & $\mathrm{X}$ \\
Acupuncture at Baihui and Dazhui & - & - & $\mathrm{X}$ & - \\
Scalp point cluster needling & - & - & - & $\mathrm{X}$ \\
\hline
\end{tabular}

TABle 2: Treatment diagram for each group.

\begin{tabular}{|c|c|c|c|c|c|c|}
\hline \multirow[b]{2}{*}{ Group } & \multicolumn{6}{|c|}{ Treatment } \\
\hline & Times/day & $\begin{array}{l}\text { Acupoint } \\
\text { selection }\end{array}$ & Needle retention duration & $\begin{array}{c}\text { Duration between } \\
\text { needle rotation } \\
\text { manipulations }\end{array}$ & $\begin{array}{l}\text { Needle rotating } \\
\text { frequency } \\
\text { r/min }\end{array}$ & $\begin{array}{c}\text { Rotation } \\
\text { manipulation } \\
\text { duration }\end{array}$ \\
\hline $\begin{array}{l}\text { Scalp point } \\
\text { cluster-needling group }\end{array}$ & 1 & $\begin{array}{l}\text { Frontal region, } \\
\text { frontoparietal } \\
\text { region, and } \\
\text { parietal region }\end{array}$ & 6 hours & 1 hour & $\begin{array}{l}\text { Approx. } \\
200 \mathrm{r} / \mathrm{min}\end{array}$ & 3 minutes \\
\hline Positive control group & 1 & Baihui, Dazhui & 1 hour & 10 minutes & $\begin{array}{l}\text { Approx. } \\
200 \mathrm{r} / \mathrm{min}\end{array}$ & 3 minutes \\
\hline Model group & \multicolumn{6}{|c|}{ None } \\
\hline Sham operation group & \multicolumn{6}{|c|}{ None } \\
\hline
\end{tabular}

2.3. Model Preparation. The permanent ligation of bilateral carotid artery method was used to create the VD rat model [8]. Feeding was stopped twelve hours prior to surgery and water was removed 4 hours prior to surgery. A $10 \%$ chloral hydrate $(3.5 \mathrm{~mL} / \mathrm{kg})$ solution was used for intraperitoneal anaesthesia. The rat was laid in a supine position and secured. An incision was made along the midline of the neck, the bilateral common carotid arteries were isolated, a number " 0 " silk thread was tied around the arteries for ligation, and the wound was closed. During surgery, the rat rectal temperature was maintained at $36.5-37.5^{\circ} \mathrm{C}$. After surgery, a penicillin sodium solution was used to coat the local area and then the rats were placed back into their cages. Penicillin sodium was injected intramuscularly at $200000 \mathrm{U} /$ rat for 3 days continuously.

2.4. Model Success Assessment. The sham operation group escape period mean was used as a reference value; if the difference between each VD rat's mean escape latency period and the reference value made up $\geq 20 \%$ of the ratio of the rat's mean escape latency period, then the model was deemed successful [9].

2.5. Animal Group Division and Management. The rats were trained in the Morris water maze for two days (after eliminating rats that could not swim or had poor scores) and randomly divided into two groups, 12 in the sham operation group and 40 in the operation group. Then, the surviving rats from the operation group (36 rats) were randomly divided into a model group, cluster-needling group, and positive control group, each containing 12 rats. Treatment was commenced 10 days after surgery, wound healing, and normal feeding.

For the scalp point cluster-needling group, the rats were first fixed on homemade metal racks; the "Laboratory Animal
Acupoint Atlas" was used to locate and needle the rat's frontal region, frontal parietal region, and parietal region (cf. human schematic diagram in Figure 1).

In each treatment region, 3 needles were inserted equal distances apart. The needles were retained for 6 hours and stimulated using the twirling method once every hour at a frequency of approximately 200 rotations per minute for 3 minutes. The rats were treated once a day for a total of 4 weeks. In the positive control group, the rats were fixed in the same way as the scalp point cluster-needling group. Baihui and Dazhui acupoints were needled, the needles were retained for 30 minutes, and needles were stimulated every 10 minutes. The frequency of rotation for stimulating Baihui was approximately 200 rotations per minute for 3 minutes, once a day, and it was carried out at the same time as the clusterneedling group. The model group and sham operation group received no acupuncture treatment. An overview of which group received which treatment is shown in Tables 1 and 2 .

\subsection{Observation Indexes and Measurements}

(a) Learning and Memory Function Measurement. After finishing the treatments, the rats were put into the water maze to test and measure each group's learning and memory function. This included directional navigation training and spatial exploration training. The time required to search for the platform and escape latency (EL) was recorded for 2 minutes.

(b) Hippocampus Tissue ACh, DA, and 5-HT Content Measurements. After the water maze testing was complete, half the rats in each group were euthanized using the spinal cord breaking method, and the brain was quickly removed, with the procedure performed on an ice bed. After the hippocampus was removed and accurately weighed, the tissue was placed in a precooled glass tube and 9 times 
TABLE 3: Comparison of water maze navigation test results for each group (mean $\pm S D$ ).

\begin{tabular}{lcr}
\hline Group & Mean escape latency period [s] & Mean swimming distance [cm] \\
\hline Sham operation group & $16.12 \pm 6.34^{* *}$ & $105.76 \pm 16.73^{*}$ \\
Model group & $115.36 \pm 13.31$ & $174.74 \pm 15.81$ \\
Positive control group & $107.52 \pm 16.11$ & $157.65 \pm 16.11$ \\
Cluster-needling group & $62.02 \pm 11.61^{* *} \triangle$ & $124.19 \pm 14.25^{* *} \Delta$ \\
\hline
\end{tabular}

Compared with the model group, ${ }^{*} P<0.05$ and ${ }^{* *} P<0.01$; compared with the positive control group, ${ }^{\triangle} P<0.05$.

the physiological saline solution was added to create a $10 \%$ brain tissue homogenate. After centrifugation, the clear fluid on top was removed and placed in a $-20^{\circ} \mathrm{C}$ refrigerator to await analyzing. The alkaline hydroxylamine colorimetric method was used to measure the ACh $[10,11]$ content in the hippocampal tissue, and fluorescence spectrophotometry was used to measure dopamine (DA) and 5-hydroxytryptamine (5-HT) content [12].

2.7. Statistical Analysis. SPSS 13.0 software was used to perform analysis of variance and the LSD test was used to compare between groups. Figure 2 shows a flowchart of the experiment in general.

\section{Results}

(a) Navigation Test. Four weeks after surgery, each group's (excluding the sham operation group) latency periods were markedly increased and swimming courses were significantly decreased. Escape latencies of rats in the model group and positive control group were significantly longer than that of the sham operation group $(P<0.01)$, indicating that VD rat learning and memory ability was decreased. The scalp point cluster- needling group rats' escape latency period was significantly shorter than that of the model group $(P<0.01)$, indicating that scalp point cluster-needling can improve VD rats' learning and memory function. There was no statistical difference between computational statistical results from the cluster-needling group and sham operation group $(P<$ 0.05). This indicates that scalp point cluster-needling can improve learning and memory function in VD model rats (see Table 3).

(b) Sham Operation Group ACh Concentration Was Significantly Higher Than That of the Model Group $(P<0.01)$. Compared with the model group, the scalp point cluster-needling group ACh concentration was significantly increased $(P<$ 0.01). The ACh concentration of the positive control group was higher than that of the model group; however, the difference was not significant, indicating that scalp point cluster-needling can increase ACh in the VD rat model, and it is more effective than regular acupuncture (see Table 4).

(c) Hippocampal DA and 5-HF Concentration Comparison between Each Group. Model rat hippocampal DA and 5-HF concentration were significantly lower than that of the sham operation group $(P<0.01)$. DA and 5 -HF concentrations in the positive control group and cluster-needling group were both higher than that of the model group, but the positive control group was not statistically significant. The clusterneedling group had a highly significant difference $(P<0.01)$ and the cluster-needling group had a statistically significant difference when compared to the positive control group $(P<0.05)$. This indicates that scalp point cluster-needling can increase VD rat hippocampal DA and 5-HF levels and its effects are better than that of regular acupuncture (see Table 4).

\section{Discussion}

Scalp point cluster-needling with long retention method was brought about by the acupuncture expert Professor Yu Zhishun [6]. It is based on his many years of clinical experience. Professor Yu Zhishun also created the "needle field" hypothesis. This new scalp acupoint differentiation method integrated field metabolism theory, central nervous system functional location on the scalp, and traditional meridian theory to divide the scalp into 7 treatment regions, including parietal region, frontoparietal region, frontal region, occipital region, lower occipital region, temporal region, and nuchal region. Presently, scalp point cluster-needling is widely used clinically, especially to treat cerebrovascular diseases, where its effectiveness is greater than that of regular scalp needling [13]. However, there is currently no information on the effectiveness of scalp point cluster-needling in treating VD.

VD is caused by cerebrovascular factors which damage brain tissue, but the exact pathogenic mechanism is still unclear. The CA1 area of the hippocampus is where the hippocampus is most closely related to human learning and memory and is easily selectively damaged by ischemia [14]. The normal function of the central cholinergic system is imperative to the formation of memories within the brain, and the cholinergic pathway is learning and memory's main pathway [15]. Within the pathway, ACh is an important neurotransmitter. VD patients' central cholinergic function is markedly decreased, which mainly is manifested in a decrease in ACh activity, causing impairment of learning and memory function [16]. Research has shown that a decrease in monoamine neurotransmitters (including DA, NH, 5-HT, etc.) is closely related to decreases in mental function in VD patients [17-19].

This experiment provides a starting point to look into the effect of scalp point cluster-needling on VD rats' hippocampal neurotransmitter levels, using a currently accepted VD animal model (permanent ligation of bilateral carotid arteries) [20]. It studies the efficacy and possible mechanisms of action of scalp point cluster-needling. The experimental 
TABLE 4: Hippocampal ACh, DA, and 5-HT measurements for each group (mean $\pm \mathrm{SD}, n=12$ per group).

\begin{tabular}{lccc}
\hline Group & ACh $[\mu \mathrm{g} / \mathrm{mg}]$ & DA $[\mathrm{ng} / \mathrm{g}]$ & 5 -HT $[\mathrm{ng} / \mathrm{g}]$ \\
\hline Sham operation group & $27.52 \pm 3.13^{* *}$ & $521.23 \pm 32.54^{* *}$ & $652.36 \pm 186.13^{* *}$ \\
Model group & $14.39 \pm 1.27$ & $362.71 \pm 43.15$ & $435.85 \pm 152.27$ \\
Positive control group & $19.05 \pm 1.98^{*}$ & $398.72 \pm 34.51^{*}$ & $525.45 \pm 147.33^{*}$ \\
Cluster-needling group & $25.82 \pm 4.61^{* * \Delta}$ & $448.63 \pm 38.62^{* * \Delta}$ & $597.59 \pm 146.35^{* * \Delta}$ \\
\hline
\end{tabular}

Compared with the model group, ${ }^{*} P<0.05$ and ${ }^{* *} P<0.01$; compared with the positive control group, ${ }^{\triangle} P<0.05$.

results indicate that while scalp point cluster-needling is improving learning and memory function in VD rats, it also significantly increases hippocampal ACh, DA, and 5HT concentrations. This indicates that scalp point clusterneedling can improve learning and memory function in VD rats, and one of its mechanisms is related to increasing neurotransmitter concentrations in the brain.

\section{Conflict of Interests}

The authors declare that there is no conflict of interests regarding the publication of this paper.

\section{Acknowledgments}

The scientific investigations were supported by the Project Research Grant (no. ZHY-Z048) from Heilongjiang Province Chinese Medicine Administration Bureau. The work was also supported by the Austrian Federal Ministries of Science, Research and Economy and of Health (project title "Evidence-Based High-Tech Acupuncture and Integrative Laser Medicine for Prevention and Early Intervention of Chronic Diseases") and the WTZ program. Professor Gerhard Litscher, Priv.-Doz. Dr. Lu Wang, and Dr. Zemin Sheng are visiting professors at the Heilongjiang University of Chinese Medicine in Harbin, China.

\section{References}

[1] M. Bomebroke and N. M. Breteler, "Epidemiology of non-AD dementias," Clinical Neuroscience Research, vol. 3, no. 6, pp. 349-361, 2004.

[2] Y. J. Wang, "Cerebral vascular disease and cognitive impairment," Chinese Journal of Internal Medicine, vol. 44, no. 11, p. 872, 2005.

[3] J. Liu and W. Z. Yang, "Progress of study on VD treated by integration of traditional and western Medicine," Chinese Journal of Integrative Medicine on Cardio-Cerebrovascular Disease, vol. 3, no. 4, p. 339, 2005.

[4] C. P. Ferri, M. Prince, C. Brayne et al., "Global prevalence of dementia: a Delphi consensus study," The Lancet, vol. 366, no. 9503, pp. 2112-2117, 2005.

[5] M. Q. Shi and W. P. Liu, "Research progress in pathogenesis of vascular dementia," Journal of the Fourth Military Medical University, vol. 28, no. 9, p. 860, 2007.

[6] B. L. Wu, H. M. Yu, and S. Q. Yang, "Professor Yu Zhishun's experience of using scalp points to treat paralysis," China Acupuncture, vol. 17, no. 3, pp. 153-154, 1997.
[7] W. Z. Zhu, J. X. Ni, Y. L. Xing et al., "The effect of scalp point cluster-needling on acute cerebral infarction patient's motor function and neurologic impairment extent," Chinese Journal of Traditional Medical Science and Technology, vol. 13, no. 2, p. 121, 2006.

[8] M. Kato, H. Iwata, T. Katayama, H. Asai, H. Narita, and T. Endo, "Possible therapeutic effect of T-794, a novel reversible inhibitor of monoamine oxidase-A, on post-stroke emotional disturbances, assessed in animal models of depression," Biological and Pharmaceutical Bulletin, vol. 20, no. 4, pp. 349-353, 1997.

[9] H. Sun and H. Lu, "Effects of butylphthalide on cognitive function, neurons of hippocampus histomorphology and the expression of nNOS in vascular dementia rats," Chinese Journal of Practical Nervous Diseases, vol. 13, no. 15, pp. 17-19, 2010.

[10] Y. Kumagae and Y. Matsui, "Output, tissue levels, and synthesis of acetylcholine during and after transient forebrain ischemia in the rat," Journal of Neurochemistry, vol. 56, no. 4, pp. 1169-1173, 1991.

[11] H. X. Yang, S. Wang, J. S. Ke et al., "Alkaline hydroxylamine colorimetric method measuring blood acetylcholine," Journal of Clinical Laboratory Science, vol. 13, no. 3, pp. 125-126, 1995.

[12] Y. Zhang, H. B. Wang, H. Yang et al., "Content determination of monoamine neurotransmitter in different encephalic regions in rat by fluorospectrophotometry," Journal of Northeast Agricultural University, vol. 41, no. 1, pp. 93-96, 2010.

[13] Q. Tang and D. S. Wang, "Effect of head acupuncture combined with facilitation techniques on movement function after stroke," Chinese Journal of Rehabilitation Theory and Practice, vol. 7, no. 2, pp. 71-72, 2001.

[14] J. Burda, M. Matiašová, M. Gottlieb et al., "Evidence for a role of second pathophysiological stress in prevention of delayed neuronal death in the hippocampal CA1 region," Neurochemical Research, vol. 30, no. 11, pp. 1397-1405, 2005.

[15] E. D. Levin and B. B. Simon, "Nicotinic acetylcholine involvement in cogitive function in animals," Psychopharmacology, vol. 138, no. 3-4, pp. 217-230, 1998.

[16] D. W. Desmond, "Vascular dementia," Clinical Neuroscience Research, vol. 3, no. 6, pp. 437-448, 2004.

[17] S. F. Xu, Neurobiology, Shanghai Medical University, Shanghai, China, 2nd edition, 1999.

[18] X. C. Ma, D. N. Zhu, Q. Wei et al., “The effect of BKHJ Chinese medicine on 6-OHDA damaged NA ascending dorsal tract of the rat brain monoamine transmitter," Shaanxi Journal of Traditional Chinese Medicine, vol. 21, no. 8, pp. 381-382, 2000.

[19] G. W. Bielenberg and M. Burkhardt, "5-Hydroxtryptamine(1A) agonists. A new therapeutic principle for stroke treatment," Stroke, vol. 21, supplement 12, pp. IV161-IV163, 1990.

[20] R. Ohtani, H. Tomimoto, T. Kondo et al., "Upregulation of ceramide and its regulating mechanism in a rat model of chronic cerebral ischemia," Brain Research, vol. 1023, no. 1, pp. 31-40, 2004. 


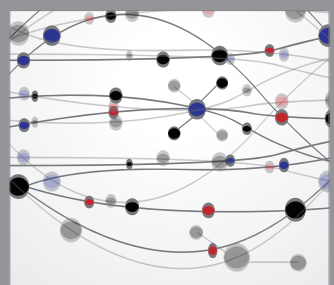

The Scientific World Journal
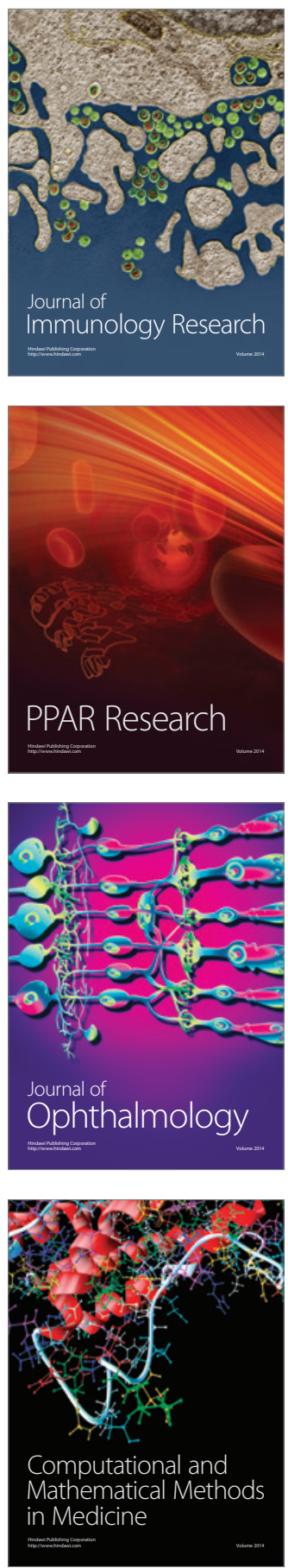

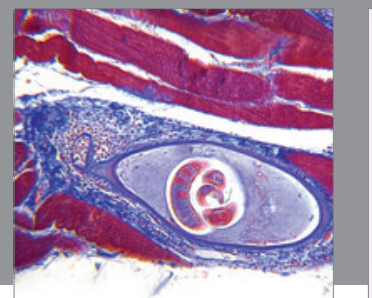

Gastroenterology

Research and Practice
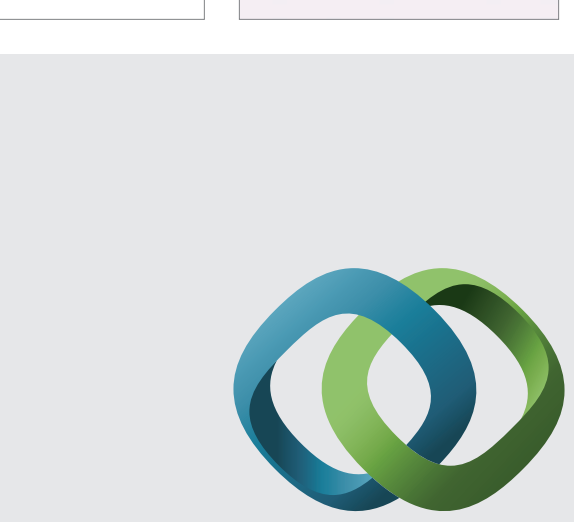

\section{Hindawi}

Submit your manuscripts at

http://www.hindawi.com
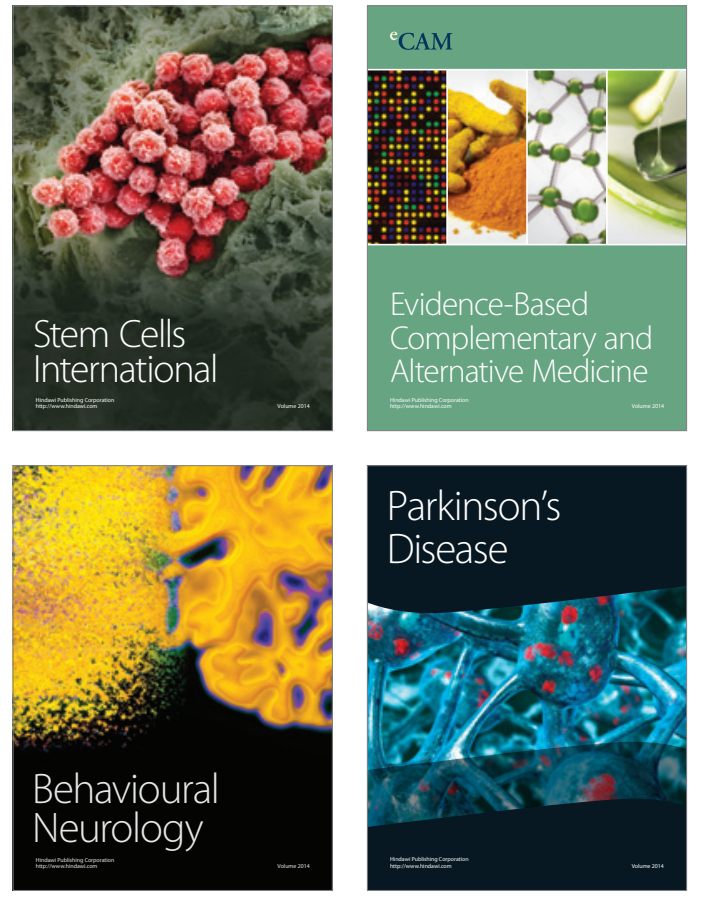
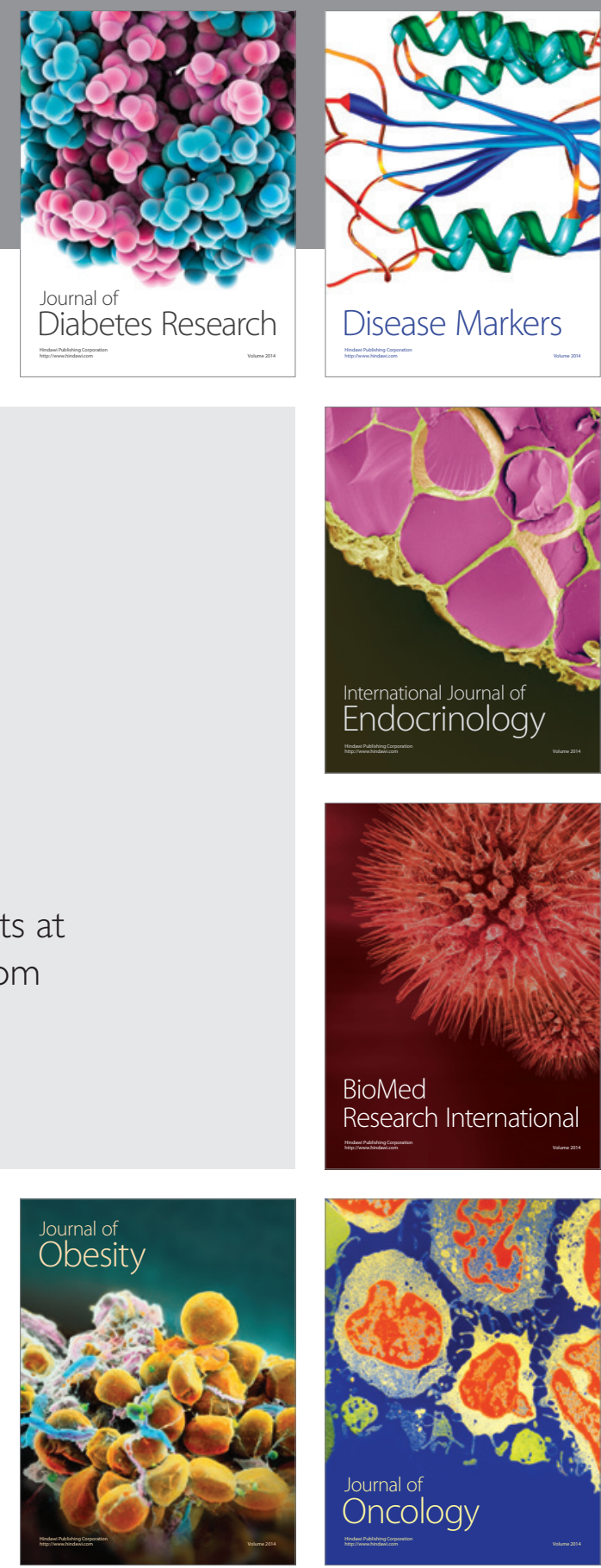

Disease Markers
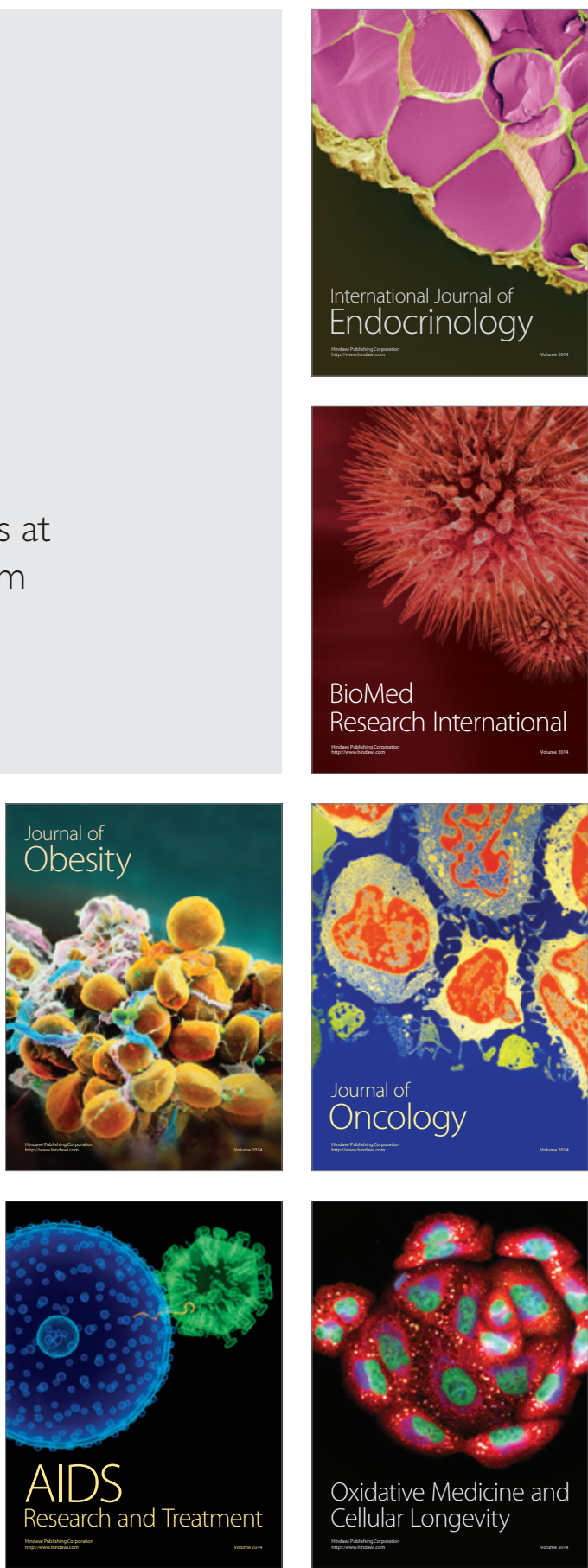